\title{
Magnetic reconnection processes induced by a CME expansion
}

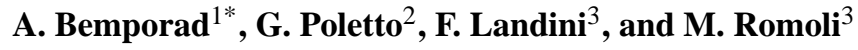 \\ ${ }^{1}$ INAF - Torino Astrophysical Observatory, via Osservatorio 20, 10025 Pino Torinese (TO), Italy \\ ${ }^{2}$ INAF - Arcetri Astrophysical Observatory, L.go E. Fermi 5, 50125 Firenze, Italy \\ ${ }^{3}$ Dept. of Astronomy and Space Science, University of Firenze, L.go E. Fermi 2, 50125 Firenze, Italy \\ *Work partially made while at the Arcetri Astrophysical Observatory, L.go E. Fermi 5, 50125 Firenze, Italy
}

Received: 1 October 2007 - Revised: 22 January 2008 - Accepted: 12 March 2008 - Published: 15 October 2008

\begin{abstract}
On 10-11 December 2005 a slow CME occurred in the Western Hemisphere in between two coronal streamers. SOHO/MDI magnetograms show a multipolar magnetic configuration at the photosphere: a complex of active regions located at the CME source and two bipoles at the base of the lateral coronal streamers. White light observations reveal that the CME expansion affects both of them and induces the release of plasma within or close to the nearby streamers. These transient phenomena are possibly due to magnetic reconnections induced by the CME expansion and occurring inside the streamer current sheet or between the CME flanks and the streamer.

These events have been observed by the SOHO/UVCS with the spectrometer slit centered at $1.8 R_{\odot}$ over about a full day. In this work we focus on the interaction between the CME and the streamer: the UVCS spectral interval included UV lines from ions at different temperatures of maximum formation such as O VI, Si XII and Al XI. These data gave us the opportunity to infer the evolution of plasma temperature and density at the reconnection site and adjacent regions. These are relevant to characterize secondary reconnection processes occurring during a CME development.
\end{abstract}

Keywords. Solar physics, astrophysics, and astronomy (Flares and mass ejections; Ultraviolet emissions) - Space plasma physics (Magnetic reconnection)

\section{Introduction}

White light images acquired in the last ten years by the Large Angle and Spectrometric COronagraphs (LASCO; Brueckner et al., 1995) on SOHO demonstrated that the occurrence of a Coronal Mass Ejection (hereafter CME) quite often is

Correspondence to: A. Bemporad

(bemporad@oato.inaf.it) followed by the formation of one or more radial features connecting the CME to the solar surface, some of them being identified as the CME flanks. Moreover, single radial white light features centered approximately at the CME latitude, connected with the expanding CME bubble and associated with EUV emission from unusually high temperature ions, have been ascribed to the post-CME current sheet (see e.g. Ciaravella et al., 2002; Ko et al., 2003; Raymond et al., 2003; Bemporad et al., 2006).

LASCO data suggest that the catastrophe loss of equilibrium can be the primary driver of CMEs (see e.g. Liu et al., 2003) and the catastrophe CME models (e.g. Lin and Forbes, 2000) predicted the formation of a current sheet connecting the top of the reconnecting post-eruption loops with the bottom of the expanding plasma bubble. However, these models start from a simple bipolar magnetic configuration, while observations indicate that the CME occurrence is associated with much more complex magnetic topologies. Starting from Antiochos et al. (1999), many quadrupolar configuration models have been developed and some recent versions of these models (see e.g. Zhang and Wang, 2007) involve the formation of two reconnecting current sheets, a transverse one above the rising flux rope and a vertical one below it (the same as in the catastrophe model). Despite the complexity reached by the actual CME models, so far little is known about the role played by nearby coronal structures (e.g. two lateral streamers in the case of a quadrupolar configuration) and in general by the overall coronal magnetic configuration in the CME occurrence, although recent UV and radio observations of flare associated CMEs suggest that the magnetic reconnection occurring between an expanding magnetic structure and nearby open fields can play a role in the eruption development (Goff et al., 2007; Démoulin et al., 2007)

In this work we focus on this subject by analyzing white light and UV observations of a CME that occurred on 10 December 2005. In particular, after a description of the

Published by Copernicus Publications on behalf of the European Geosciences Union. 


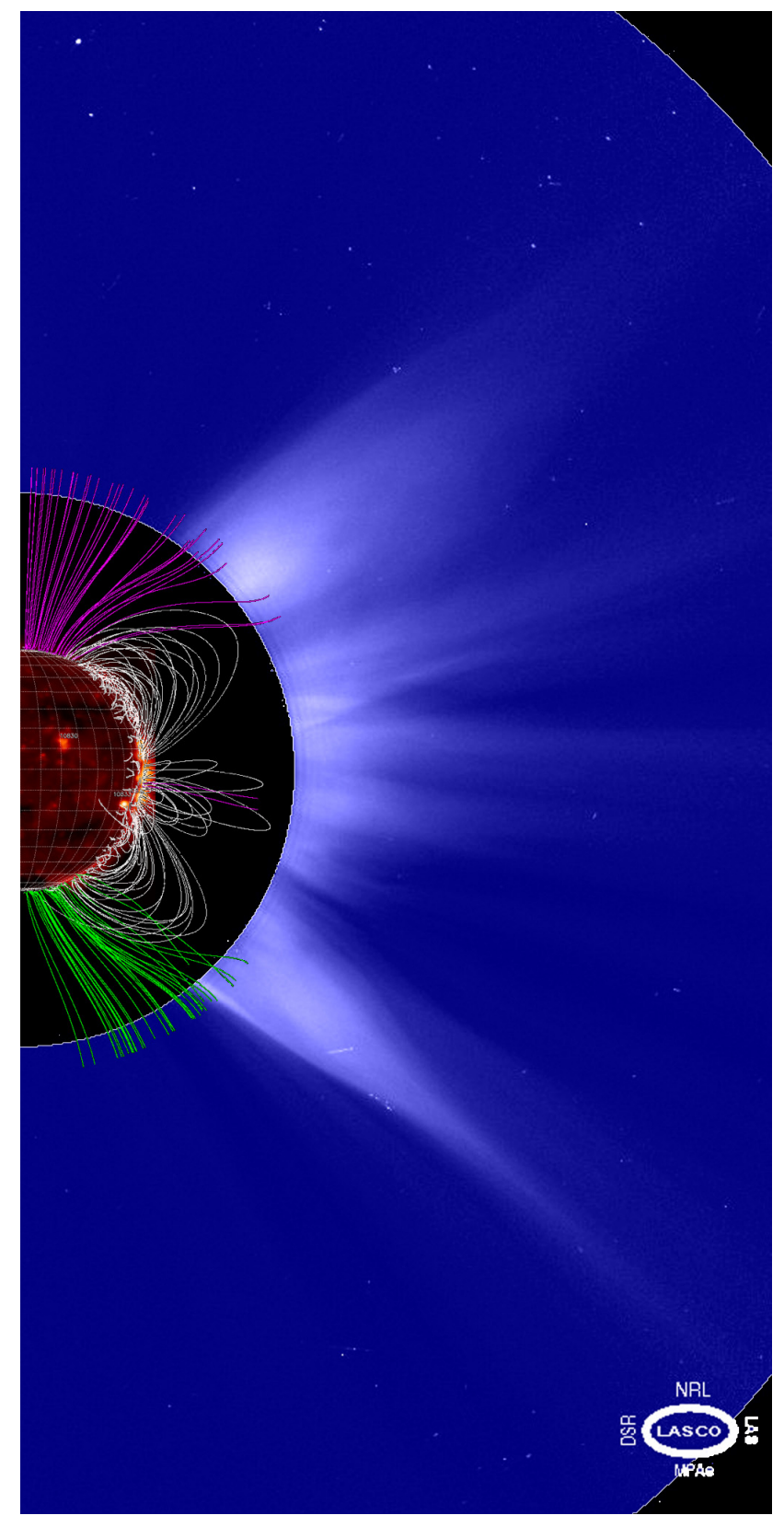

Fig. 1. The white light solar corona (west limb) as seen by the SOHO/LASCO C2 coronagraph before the CME (10 December 13:00 UT) and a soft X-ray image of the solar disk made by the GOES/SXI instrument (10 December 12:58 UT). Superposed open (green and purple) and closed (white) fieldlines have been computed with a PFSS extrapolation (see text) from Carrington rotation map 2037.

photospheric and coronal magnetic fields (Sect. 2), we discuss from white light images acquired above $2 R_{\odot}$ how the propagation of the CME induced outflows along the nearby coronal streamers (Sect. 3). Hence, we show how the same phenomena have been observed at $1.8 R_{\odot}$ in the UV lines by the UV Coronagraph Spectrometer (UVCS; Kohl et al., 1995) on SOHO; from these data we derive the evolution of plasma physical parameteres close to the streamer during magnetic reconnection (Sect. 4). Discussion of our results and conclusions are given in the last section (Sect. 5).

\section{The ambient solar corona}

The white light images of the solar corona acquired by the LASCO/C2 coronagraph show on 10 December 2005 the presence of two streamers centered at an approximate latitude of $39^{\circ} \mathrm{NW}$ and $40^{\circ} \mathrm{SW}(18: 00 \mathrm{UT})$ and a complex of smaller radial structures in between (Fig. 1). On the same day, X-ray images of the solar disk show that a system of active regions (ARs NOAA 10826, 10827, 10828, 10829, 10831), located close to the equator (latitudes between $15^{\circ} \mathrm{N}$ and $10^{\circ} \mathrm{S}$ ), is crossing the West solar limb; this indicates that the white light structures observed between the two streamers are related to these ARs (Fig. 1).

The configuration of coronal magnetic fields projected onto the plane of the sky on 10 December 2005 ( 12:00 UT) is shown in Fig. 1 (Carrington Rotation map nbr. 2037); coronal magnetic fields have been computed starting from CR map of photospheric fields made by MDI and using a Potential Field Source Surface (PFSS) extrapolation. This figure shows that, despite the complex X-ray morphology visible on the solar disk, the magnetic fields along the meridian at the solar limb between 10-11 December have a well defined quadrupolar configuration. In particular, the extrapolation shows two groups of extended closed loops (associated with the northwest and southwest streamers in white light images) and a more confined system of loops at the equator in correspondence of the equatorial AR's. As we describe in the next section, late on 10 December 2005 a CME is launched from the equatorial region at the West limb: hence we may expect some interactions between the expanding plasma bubble associated with the CME and the two coronal streamers. Before concluding this section we note here that the configuration shown in Fig. 1 differs from those envisaged in quadrupolar models by the absence of a transverse current sheet connecting the cusps of the two streamers: in our case the existence of this current sheet will not be in agreement with the configuration shown by LASCO images.

\section{The 10-11 December 2005 events}

The coronal configuration before our event is shown again in Fig. 2 (panel a); starting on 19:00 UT, a CME appeared at the West limb in the LASCO/C2 field of view. LASCO/C2 difference images show a complex of opening nested loops followed by the superposition of many filamentary pattern probably linked to the loop footpoints down in the photosphere (Fig. 2, panel b). This slow CME $(\sim 50 \mathrm{~km} / \mathrm{s}$ around 
$3 R_{\odot}$ ) expanded at a latitude of about $10^{\circ} \mathrm{SW}$ with an angular width of $\sim 70^{\circ}$ : hence the CME propagated in between the two streamers, with its flanks close to the streamer axes.

During the CME development LASCO images show the occurrence of other interesting phenomena: starting from about 03:00 UT on 11 December the northwest streamer appears to pinch off and the propagation of a concave Y-shaped feature along the streamer axis is observed (Fig. 2, panels bc). In the following hours a similar process occurs also in the southwest streamer that clearly shows the formation of a Xpoint in between two radial features approaching each other, the northward one being aligned with the CME southward flank. The X-point stretches in two Y-shaped features, one propagating outward along the streamer and the other rooted on the Sun, while a current sheet develops in between (Fig. 2, panel e). About $12 \mathrm{~h}$ later the pre-CME coronal configuration is mainly recovered (two streamers at intermediate latitude), but the equatorial region is less bright in white light likely because of the density decrease in the region where the CME occurred (Fig. 2, panel f).

Interestingly, the formation of the outward propagating Yshaped feature in the south streamer is preceeded by a secondary small ejection occurring northward of the streamer boundaries around $\sim$ 12:00 UT (Fig. 2, panel d). A deeper study of this small event shows that it consist of an unstructured eruption including many different blobs propagating at different speeds (Fig. 3). From LASCO/C2 difference images we concluded that in this small eruption the outflow speeds span from $\sim 40 \mathrm{~km} / \mathrm{s}$ (feature indicated by a red arrow in Fig. 3) up to $\sim 250 \mathrm{~km} / \mathrm{s}$ (feature indicated by a blue arrow in Fig. 3). LASCO difference images show clearly this secondary eruption to arise from a region comprised in between the southward expanding CME flank and the streamer boundary and to propagate mainly parallel to the CME flank. The correspondence between the orientation of the southward CME flank and the latitude of the secondary eruption and of the northward approaching feature forming later the $\mathrm{X}$-point suggests that these events are probably related to the CME expansion.

The northwest streamer event has been already studied by Wang and Sheeley (2006): the authors found from LASCO images that this streamer is slowly expanding in the days before 11 December and that this expansion leads to the observed detachment. As pointed out by these authors, EIT and MDI data show the presence of a bipolar magnetic region located below the northwest streamer and the emergence of several smaller ARs located mainly around the equator. Wang and Sheeley (2006) concluded that this flux emergence drives the streamer expansion until, via magnetic reconnection in the expanding loop arcade, a non-equilibrium configuration is reached leading to the observed detachment. However, looking at LASCO movies it is evident that the CME and the streamer detachment occur together: hence it is likely that, at the end of the expansion described by Wang and Sheeley (2006), the occurrence of the CME at the side of

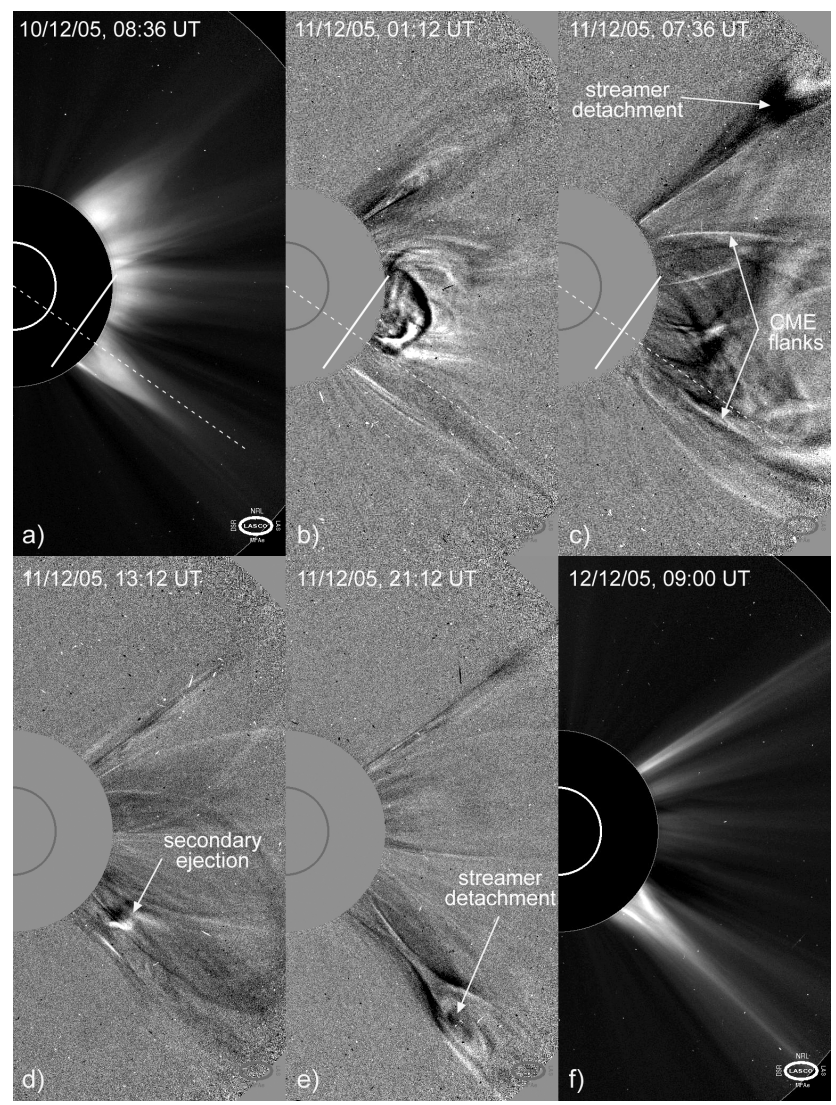

Fig. 2. The white light solar corona (west limb) as seen by the LASCO/C2 coronagraph in standard (panels a and f) and running difference (panels b-e) images; panel (a): coronal configuration before the event; panel (b): CME propagation; panel (c): northwest streamer detachment; panel (d): secondary ejection after the CME; panel (e): southwest streamer detachment; panel (f): coronal configuration after the events; observation times are indicated in each panel. The solid white line corresponds to the position of the UVCS slit field of view, while the dashed line shows the radial towards the slit center at a latitude of $35^{\circ} \mathrm{S}$; UVCS observations respectively started and ended rougthly at times corresponding to panels (a) and (c).

the streamer accelerates this process helping the reconnection inside the streamer and destabilizing the overall magnetic configuration. Although similar to this, the interpretation we propose for the events occurring southward of the CME is not identical because we have to explain not only the streamer detachment, but also the secondary eruption.

From the above considerations, taking also into account the coronal magnetic configuration described above, we tentatively explain the overall evolution as follows: the emergence of magnetic flux in the equatorial region leads to an unstable configuration that erupts as a CME and induces at the same time the progressive expansion of the northwest streamer observed by Wang and Sheeley (2006). Hence, 


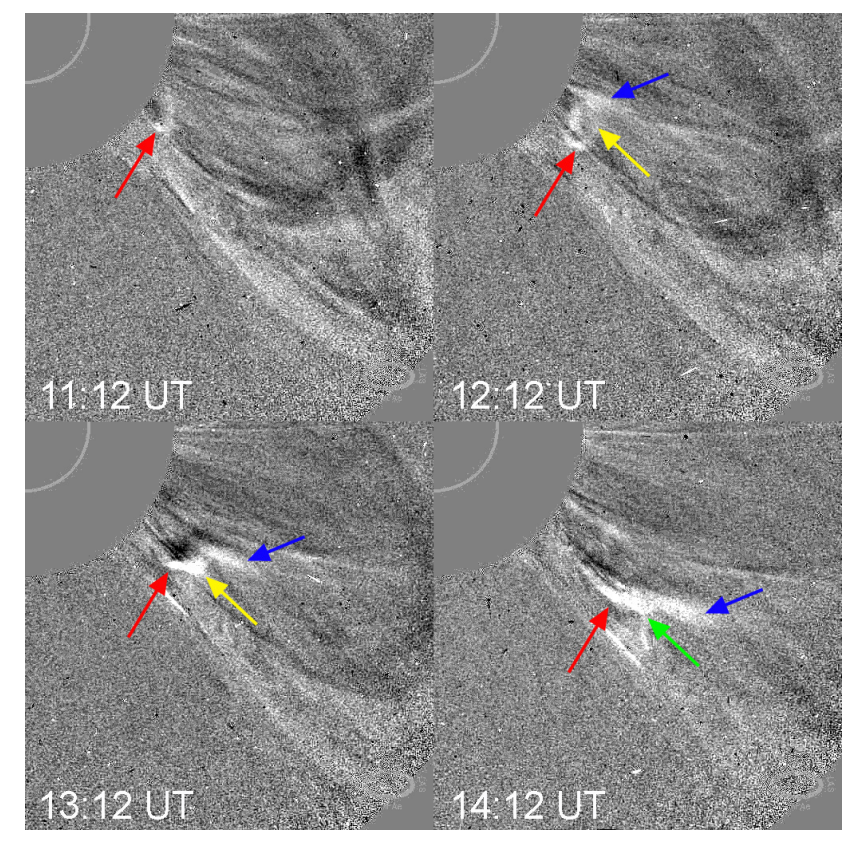

Fig. 3. LASCO/C2 difference images showing the white light evolution during the secondary eruption. From these images we estimated the outflow speeds of different features (tentatively identified with arrows of different colors) that turn out to be between $\sim 40 \mathrm{~km} / \mathrm{s}$ (red arrowed feature) and $\sim 250 \mathrm{~km} / \mathrm{s}$ (blue arrowed feature).

the northward and southward expanding CME flanks interact with lateral streamers pushing the oppositely directed fieldlines against each other and inducing magnetic reconnection. In the northwest streamer the reconnection is induced along the streamer current sheet leading to the observed detachment along the streamer axis. In the coronal region located southward of the CME the situation is more complicated, as suggested by LASCO difference images. As the CME propagates outward magnetic reconnection occurred first between the expanding CME flank and the streamer boundaries, producing the observed secondary eruption (Fig. 1, panel $\mathrm{d}$ and Fig. 4, panels 1-4). Different blobs propagating at different speed are probably produced by magnetic reconnection processes occurring at various altitudes and latitudes during the expansion of the CME flanks towards the streamer boundaries. Later on, the propagation of the secondary eruption close to the southwest streamer axis induces the magnetic reconnection along the streamer current sheet, leading to the formation of the two Y-shaped features described above that, accelerated by magnetic tension, propagate outward generating the streamer detachment (Fig. 1, panel e and Fig. 4, panels 5-8). As explained in the next section, this interpretation is also supported by the evolution of the UV line intensities observed during this event by UVCS below the LASCO/C2 occulter.

\section{UVCS observations}

The position of the UVCS slit field of view is shown in Fig. 2 (panels a-c): in particular the slit was centered at a latitude of $35^{\circ} \mathrm{S}$ in the West limb and covered approximately a latitude interval between $3^{\circ} \mathrm{N}$ and $64^{\circ} \mathrm{S}$, hence the slit was favourably located to observe both the CME and the evolution of the southwest streamer. UVCS observed the corona with a spatial resolution of $42 \operatorname{arcsecs}\left(\sim 3 \times 10^{4} \mathrm{~km}\right)$ and a time resolution of $2 \mathrm{~min}$ (exposure time) over a $\sim 23 \mathrm{~h}$ time interval between 10 December, 08:29 UT (Fig. 2, panel a) and 11 December, 07:33 UT (Fig. 2, panel c). The selected spectral intervals included the O VI $\lambda \lambda 1032-1037 \AA$ doublet lines, the H Lyman- $\beta \lambda 1025 \AA$ and Lyman- $\gamma \lambda 973 \AA$ lines and the Si XII $\lambda 520 \AA$, [Al XI] $\lambda 550 \AA$ second order lines. During the observations the grating position has been changed, so that in the last dataset $(\sim 7 \mathrm{~h}$ from 11 December, 00:23 to 07:33 UT) we observed the [Al XI] $\lambda 550 \AA$ (non included in the previous datasets), but we did not observe the O VI $\lambda 1037$ and Si XII $\lambda 520 \AA$ lines. The [Fe XIII] $\lambda 510 \AA$ (second order) and $[\mathrm{Fe} \mathrm{X}] \lambda 1028 \AA$ lines were also included in the selected spectral intervals, but no significant emission was detected in these lines.

In order to increase the signal to noise ratio we averaged these data over 2 spatial bins (reducing the spatial resolution to $\sim 6 \times 10^{4} \mathrm{~km}$ ) and 10 exposures (reducing the temporal resolution to $\sim 20 \mathrm{~min}$ ). By integrating each spectral line over the observed profile and subtracting the average background we computed the evolution of line intensities at different spatial bins along the slit. The resulting intensity evolution of O VI $\lambda 1032 \AA$ is shown in Fig. 5 (panel a). At the beginning of the UVCS observations the southwest streamer was centered approximately at a latitude of $\sim 40^{\circ} \mathrm{S}$, hence $5^{\circ}$ or $\sim 10^{5} \mathrm{~km}$ from the slit center. Hence Fig. 5 (panel a) shows that during our observations the main UV emission is associated with the southwest streamer and that apparently there is no significant variation of the line intensity. In order to highlight minor intensity variations (larger than the statistical uncertainties) we show in Fig. 5 the O VI $\lambda 1032 \AA$ line intensity evolution after subtracting from each bin along the slit its intensity averaged over 1) the first 20 exposures of observation (i.e. $40 \mathrm{~min}$; Fig. 5, panel b) and 2) the whole observation interval ( $\sim 23 \mathrm{~h}$; Fig. 5, panel c). Both panels (b) and (c) show a more complex pattern: in particular, it is possible to recognize the CME front and void, while the identification of the CME core is more uncertain. Interestingly, these panels show that, during the CME propagation, two approaching features are visible close to the streamer core: two branches, initially at a relative distance of $\simeq 3.6 \times 10^{5} \mathrm{~km}$ in panel (c), start approaching each other $\sim 5 \mathrm{~h}$ after the UVCS observations start. The two branches, both moving towards the center of the streamer, coalesce $\sim 11 \mathrm{~h}$ after the UVCS observation start (hence on 11 December, 00:30 UT), as we inferred by defining the "coalescence time" as the time the $\mathrm{O}$ VI emission peaks (see later). 


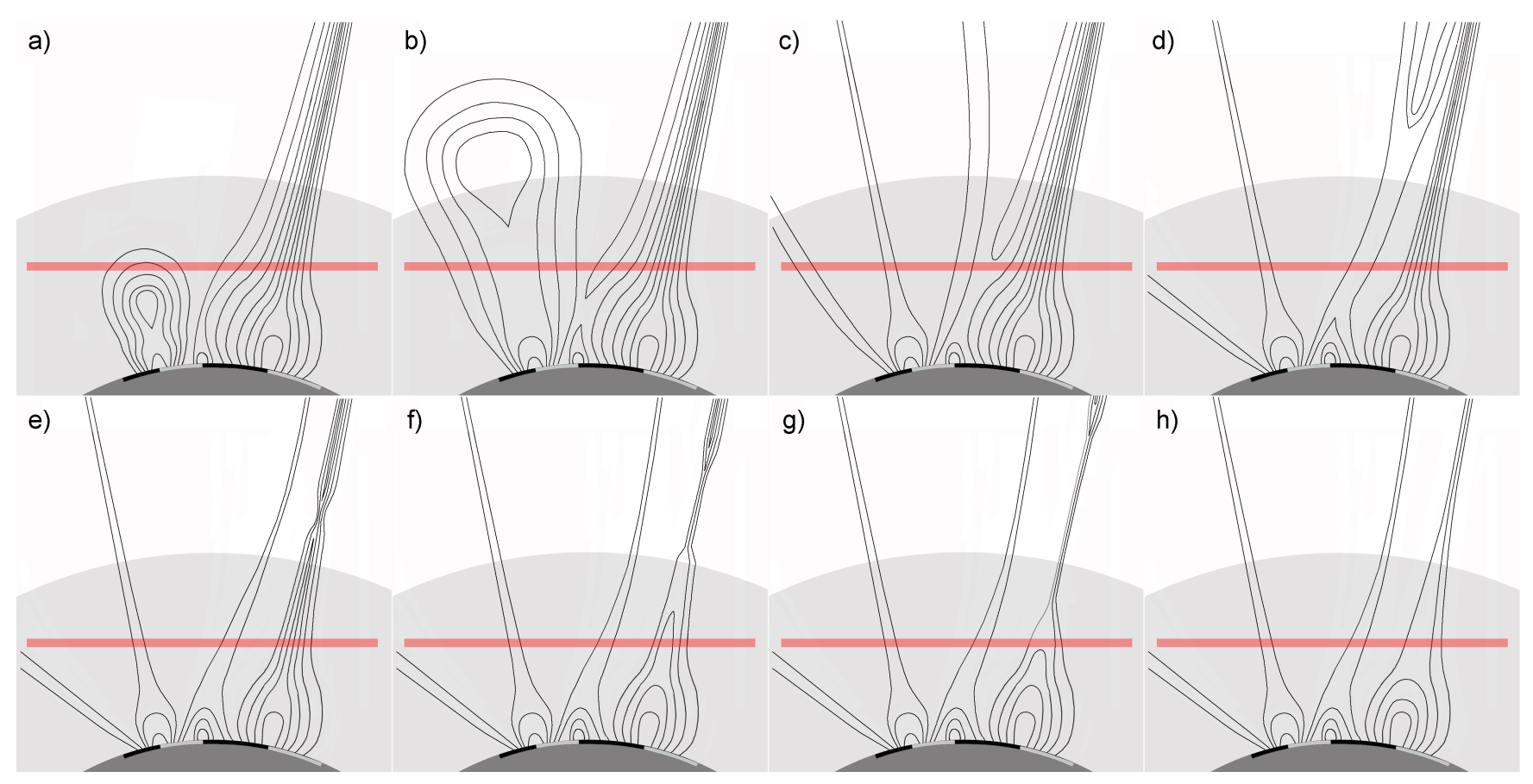

Fig. 4. A cartoon showing the interaction between the expanding CME and the southwest streamer: positive and negative polarities are shown as light and dark gray areas respectively; shaded regions represent coronal altitudes located below the LASCO/C2 occulter (gray) and in the UVCS field of view (red); dimensions are not to scale. As the CME expands (panel a) magnetic reconnection is induced between the CME flank and the streamer (panel b); this reconnection proceeds towards higher levels leading finally to the secondary eruption (panels $\mathbf{c}$ and $\mathbf{d}$ ). The propagation of the eruption close to the streamer axis leads to the streamer pinching by magnetic reconnection along the streamer current sheet (panel $\mathbf{e}$ ) and to the subsequent formation of the two Y-shaped features (panel f) and the final release of a streamer detachment (panels $\mathbf{g}$ and $\mathbf{h}$ ).
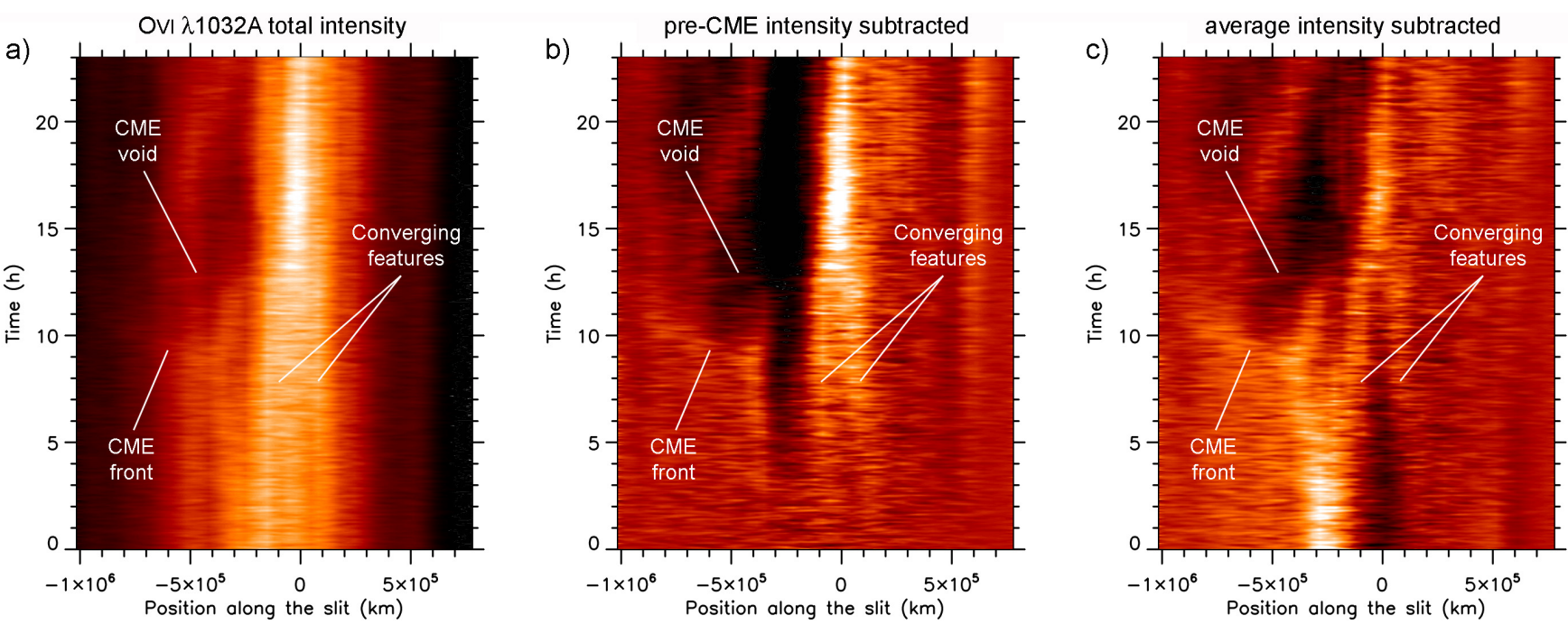

Fig. 5. The evolution of O VI $\lambda 1032 \AA$ total intensity (a) and of the line intensity after the subtraction of the average pre-CME values (b) and of the average over the whole observation interval (c). Time in the $y$ axes is given starting on 10 December, 08:29 UT, while the " 0 " in the $\mathrm{x}$-axes marks the position of the slit center. Colors range from 0 (black) to $3 \times 10^{10}$ phot $\mathrm{cm}^{-2} \mathrm{~s}^{-1} \mathrm{sr}^{-1}$ (white) in panel a and from $-6 \times 10^{9}$ (black) to $+6 \times 10^{9}$ phot $\mathrm{cm}^{-2} \mathrm{~s}^{-1} \mathrm{sr}^{-1}$ (white) in panels (b) and (c). 

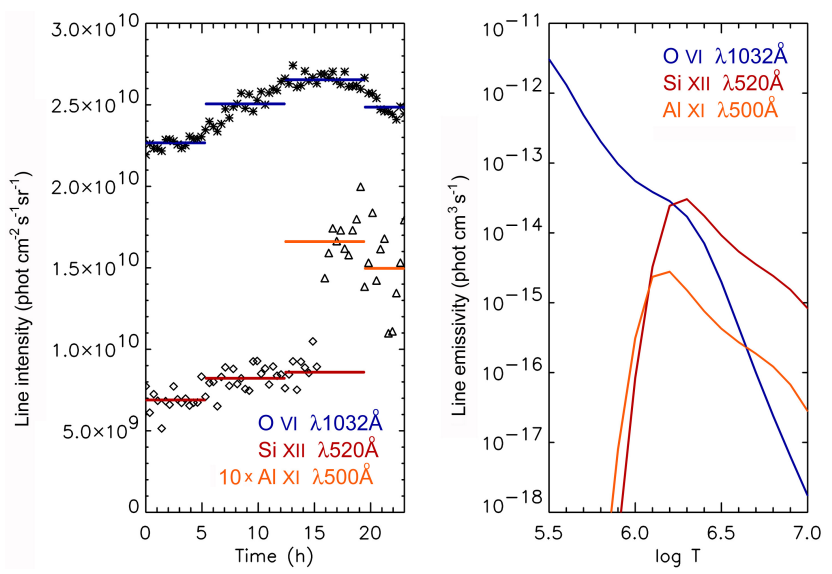

Fig. 6. Left panel: the intensity of O VI $\lambda 1032 \AA$ (stars), Si XII $\lambda 520 \AA$ (triangles) and $[\mathrm{Al} \mathrm{XI}] \lambda 550 \AA$ (boxes) averaged over the latitudes of the reconnecting region; solid horizontal lines show the average intensity values used to estimate plasma parameters in four time intervals (see text). Right panel: O VI $\lambda 1032 \AA$ (blue), Si XII $\lambda 520 \AA$ (red) and [Al XI] $\lambda 550 \AA$ (orange) line emissivities as a function of plasma temperature.

\section{UVCS data analysis and interpretation}

A comparison between the evolution observed in white light images by LASCO starting on 10 December, 19:00 UT above $2 R_{\odot}$ (see Figs. 2 and 3 ) and that of the EUV emission at earlier times below this altitude suggests that the coalescence of EUV branches and the white light secondary eruption are related. In our interpretation, the EUV coalescence observed close to the streamer core is driven by the magnetic reconnection between the CME flanks and the streamer boundaries. This reconnection is induced by the CME expansion occurring first below the altitude of the UVCS field of view $\left(1.8 R_{\odot}\right.$; Fig. 4 , panel $\left.\mathrm{b}\right)$; during the expansion of the main $\mathrm{CME}$ the reconnecting $\mathrm{X}$-point raises with time crossing the UVCS slit field of view (Fig. 3, panel c) and finally leading to a multi-structured secondary eruption that escapes from the Sun as observed by LASCO/C2 (Figs. 3 and 4, panel d). The transit of the reconnecting X-point across the slit field of view corresponds to the observed coaleshence of two branches (Fig. 5) that results in the formation of a single radial feature located close to the streamer (Fig. 4, panels d-e). Over the following hours the propagation of the secondary CME close to the streamer triggered the magnetic reconnection along its current sheet (Fig. 2 panel e and Fig. 4, panels $\mathrm{f}-\mathrm{g}$ ) and the observed streamer detachment. We note here that the coalescence of two converging features described above cannot be explained as a simple compression and deflection of the streamer during the CME expansion. Figure 5 demonstrates the presence of quasi symmetric inflows from both sides towards the same region and the CME expansion cannot be responsible for plasma motions towards the CME itself, unless magnetic reconnection is occurring.

In the following we concentrate on the EUV line intensity evolution observed at the latitude where the reconnection occurred: starting from intensity panels shown in Fig. 5, in order to increase the statistical significance of the data, we averaged the observed emission over a 6 spatial bins $\left(\sim 1.8 \times 10^{5} \mathrm{~km}\right)$ interval centered on the reconnecting region. The resulting average evolution of O VI $\lambda 1032 \AA$, Si XII $\lambda 520 \AA$ and $[\mathrm{Al} \mathrm{XI}] \lambda 550 \AA$ lines is shown in Fig. 6 (left panel) together with the line emissivities (right panel) from the CHIANTI ${ }^{1}$ spectral code (v. 5.2). Over the observed O VI intensity evolution we identified in the whole process four time intervals: a pre-reconnection interval (hereafter time interval 1), a reconnection interval (2), a main phase (3) and a post-reconnection (4) interval. These correspond respectively to periods where, in first approximation, the $\mathrm{O}$ VI intensity is constant (1), increases (2), is approximately constant around the maximum (3) and decreases (4). The resulting average intensities over these time intervals are shown in Fig. 6 as horizontal lines.

Average values of the O VI $\lambda \lambda 1032-1037 \AA$, Si XII $\lambda 520 \AA$ and $[\mathrm{Al} \mathrm{XI}] \lambda 550 \AA$ line intensities in these four time intervals have been used to estimate the plasma parameters inside the reconnecting region before, during and after the magnetic reconnection. This has been done as follows: first, in order to isolate the UV emission coming from the reconnecting region, we computed the contribution of the plasma external to this region and aligned along the line of sight to the observed intensity. To this end, we estimated the average coronal density profile from daily LASCO $\mathrm{pB}$ images (using the standard $\mathrm{pB}$ inversion technique in the crude hypothesis of a spherically symmetric corona). By assuming outflow speeds from a simple mass flux conservation, coronal temperatures given by Gibson et al. (1999), line emissivities from the CHIANTI spectral code and streamer elemental abundances given by Bemporad et al. (2003), we estimated the external coronal contribution to the total O VI, Si XII and [Al XI] line intensities by integrating the line emissivities along the entire line of sight (excluding a region centered on the plane of the sky with extension along the line of sight equal to the projected southwest steramer extension). By subtracting these values to the observed intensities we isolated the emission of the reconnecting region.

Then, intensities of O VI $\lambda \lambda 1032-1037 \AA$ lines have been used to derive plasma densities with the standard technique that makes use of the ratio between the collisional and radiative components of the O VI $\lambda 1032 \AA$ line (Noci et al., 1987). This technique, typically used in the hypothesis of a static plasma, has been recently modified by Antonucci et al. (2005) to remove this hypothesis and take also the Doppler dimming effect into account. Recently it has been suggested (Noci and Gavryuseva, 2007) that slow outflows are possible

\footnotetext{
${ }^{1}$ See http://wwwsolar.nrl.navy.mil/chianti.html.
} 
below the altitude of $2 R_{\odot}$ along the boundaries of solar minimum coronal streamers. Hence, in our computation we assumed that during the time interval 1 the plasma in the reconnecting region located at the streamer boundary is outflowing along the pre-reconnection open fieldlines at $\sim 50 \mathrm{~km} / \mathrm{s}$, in the intervals 3 and 4 the outflow is negligible, while in interval 2 we assumed an intermediate speed of $\sim 30 \mathrm{~km} / \mathrm{s}$. Densities derived with this technique also depend on the unknown electron temperature $T_{e}$ : hence, assuming Bemporad et al. (2003) streamer elemental abundances, we computed for different temperatures the expected intensity of O VI, Si XII and [Al XI] lines and we selected in each time interval the $T_{e}$ value that better reproduces the observed intensities. Results from this technique are described and discussed in the next section.

\section{Conclusions}

Densities and temperatures inside and outside the reconnecting region are given in Table 1; in particular we conclude that before the CME this region was denser $(\sim+60 \%)$ and hotter $(\sim+50 \%)$ than the surrounding corona. Then, during the reconnection process, density and temperature increase respectively by $\sim 20 \%$ and $\sim 30 \%$; the results for the last time interval are only tentative because the O VI $\lambda 1037 \AA$ line was not included in the selected spectral range and the separation between radiative and collisional components of the O VI $\lambda 1032 \AA$ (needed to derive densities) has been done by assuming a constant $I(1032) / I(1037)$ ratio. The observed temperature increase can be due to the plasma that, pushed towards the streamer by the CME expansion, is heated by an adiabatic compression. In this case the final temperature $T_{f}$ (in interval 3 ) reached by the compressed plasma would be

$T_{f}=T_{i} \cdot\left(\frac{n_{i}}{n_{f}}\right)^{1-\gamma} \simeq 2.0 \times 10^{6} \mathrm{~K}$

where $n_{f}\left(\mathrm{~m}^{-3}\right)$ is the electron density at the end of the reconnection process and $n_{i}, T_{i}$ are the electron density and temperature before the reconnection starts (see values in Table 1). This final temperature is slightly smaller than that we determined, but the difference is below the statistical uncertainties associated with our estimates. We may conclude that the plasma heating provided by the conversion of magnetic to thermal energy via reconnection is, if any, negligible: adiabatic compression heating dominates. This can be explained by taking into account that the observed reconnection occurred at high levels in the corona $\left(1.8 R_{\odot}\right)$ where the magnetic fields are weak. However, starting from a typical coronal magnetic field of $\sim 1-0.1 \mathrm{G}$, the density of magnetic energy is $u_{m}=B^{2} /(2 \mu)=4 \times 10^{-3}-4 \times 10^{-5} \mathrm{~J} \mathrm{~m}^{-3}$; this value is comparable with the observed variation of gas internal energy density, which is given by

$\Delta e=3 K\left(n_{f} T_{f}-n_{i} T_{i}\right) \simeq 3 \times 10^{-4} J^{-3}$
Table 1. Plasma parameters inside the reconnecting region.

\begin{tabular}{ccc}
\hline Interval & $T_{e}(\mathrm{~K})$ & $n_{e}\left(\mathrm{~m}^{-3}\right)$ \\
\hline Pre-rec. & $1.8 \times 10^{6}$ & $6.5 \times 10^{12}$ \\
Rec. & $2.2 \times 10^{6}$ & $7.2 \times 10^{12}$ \\
Main & $2.5 \times 10^{6}$ & $7.6 \times 10^{12}$ \\
Post-rec. & $>2.5 \times 10^{6}$ & $>1.0 \times 10^{13}$ \\
Corona & $1.2 \times 10^{6}$ & $4.0 \times 10^{12}$ \\
\hline
\end{tabular}

This implies that magnetic fields close to the streamer boundary have to be lower than $1 \mathrm{G}$.

In conclusion, in this work we had the unique opportunity to study the evolution of plasma parameters inside a reconnecting region: we point out that this is the first time that a reconnection process has been directly observed within the UVCS field of view as the progressive merging of two features. As we have shown, this process has been induced by the nearby expansion of a CME: this may suggest that the interaction of CMEs with extended coronal structures could also play a role in the overall evolution of these events. In the future we plan also to analyze the evolution of UV lines inside the CME in order to derive informations on the plasma physical parameters at the CME front and void. Moreover, from the study of geometrical properties of the evolving current sheet in white light during the streamer detachment we plan also to derive informations on the reconnection rate and to compare this parameter with values predicted by $\mathrm{CME}$ models.

Acknowledgements. A. Bemporad and G. Poletto thank ISSI (International Space Science Institute, Bern) for the hospitality provided to the members of the team on the Role of Current Sheets in the Solar Eruptive Phenomena, where many ideas presented in this work have been discussed. A. Bemporad and G. Poletto acknowledge support from ASI/INAF I/05/07/0 and I/035/05/0.

Topical Editor R. Forsyth thanks two anonymous referees for their help in evaluating this paper.

\section{References}

Antiochos, S. K., DeVore, C. R., and Klimchuk, J. A.: A Model for Solar Coronal Mass Ejections, Astrophys. J., 510, 485-493, 1999.

Antonucci, E., Abbo, L., and Dodero, M. A.: Slow wind and magnetic topology in the solar minimum corona in 1996-1997, Astron. Astrophys., 435, 669-711, 2005.

Bemporad, A., Poletto, G., Suess, S. T., et al.: Current Sheet Evolution in the Aftermath of a CME Event, Astrophys. J., 638, 1110 1128, 2006.

Bemporad, A., Poletto, G., Suess, S. T., et al.: Temporal Evolution of a Streamer Complex: Coronal and in Situ Plasma Parameters, Astrophys. J., 593, 1146-1163, 2003. 
Brueckner, G. E., Howard, R. A., Koomen, M. J., et al.: The Large Angle Spectroscopic Coronagraph (LASCO), Solar Phys., 162, 357-402, 1995.

Ciaravella, A., Raymond, J. C., Li, J., et al.: Elemental Abundances and Post-Coronal Mass Ejection Current Sheet in a Very Hot Active Region, Astrophys. J., 575, 1116-1130, 2002.

Démoulin, P., Klein, K.-L., Goff, C. P., et al.: Decametric N Burst: A Consequence of the Interaction of Two Coronal Mass Ejections, Solar Phys., 240, 301-313, 2007.

Gibson, S. E., Fludra, A., Bagenal, F., et al.: Solar minimum streamer densities and temperatures using Whole Sun Month coordinated data sets, J. Geophys. Res., 104, 9691-9700, 1999.

Goff, C. P., van Driel-Gesztelyi, L., Démoulin, P., et al.: A Multiple Flare Scenario where the Classic Long-Duration Flare Was Not the Source of a CME, Solar Phys., 240, 283-299, 2007.

Ko, Y.-K., Raymond, J. C., Lin, J., et al.: Dynamical and Physical Properties of a Post-Coronal Mass Ejection Current Sheet, Astrophys. J., 594, 1068-1084, 2003.

Kohl, J. L., Esser, R., Gardner, L. D., et al.: The Ultraviolet Coronagraph Spectrometer for the Solar and Heliospheric Observatory, Solar Phys., 162, 313-356, 1995.
Lin, J. and Forbes, T. G.: Effects of reconnection on the coronal mass ejection process, J. Geophys. Res., 105, 2375-2392, 2000.

Liu, Y., Jiang, Y.-C., Ji, H.-S., et al.: Observational Evidence of a Magnetic Flux Rope Eruption Associated with the X3 Flare on 2002 July 15, Astrophys. J., 593, L137-L140, 2003.

Noci, G., Kohl, J. L., and Withbroe, G. L.: Solar wind diagnostics from Doppler-enhanced scattering, Astrophys. J., 315, 706-715, 1987.

Noci, G. and Gavryuseva, E.: Plasma Outflows in Coronal Streamers, Astrophys. J., 658, L63-L66, 2007.

Raymond, J. C., Ciaravella, A., Dobrzycka, D., et al.: FarUltraviolet Spectra of Fast Coronal Mass Ejections Associated with X-Class Flares, Astrophys. J., 597, 1106-1117, 2003.

Wang, Y.-M. and Sheeley, N. R.: Observations of Flux Rope Formation in the Outer Corona, Astrophys. J., 650, 1172-1183, 2006.

Zhang, Y.-Z. and Wang, J.-X.: A Catastrophic Flux Rope in a Quadrupole Magnetic Field for Coronal Mass Ejections, Astrophys. J., 663, 592-597, 2007. 